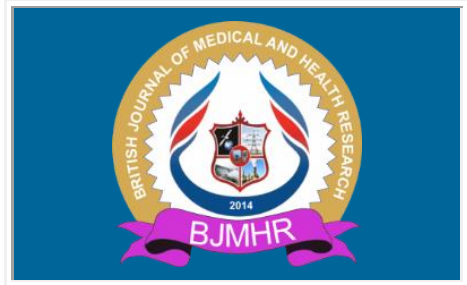

\title{
BJMHR
}

British Journal of Medical and Health Research

Journal home page: www.bjmhr.com

\section{COVID19 and Repurposing of Drugs for Prophylaxis}

\section{Mrudula Phadke ${ }^{1 *}$, Sujata Saunik ${ }^{2}$ \\ 1 Sr. Adv, NHM, Government of Maharashtra, UNICEF, Mumbai, Ex.VC Maharashtra University of Health Sciences, Maharashtra, India. Orcid id: https://orcid. org/0000-0002-6441-9623 \\ 2 A.C. Sec I.A.S. Govt of. Maharashtra, Mumbai, Harvard T.H. Chan School of Public Health, Harvard University, Boston, Massachusetts, USA \\ Orcid id: https://orcid.org/0000-0001-6641-0721}

\section{ABSTRACT}

Corona virus disease (COVID-19) has created pandemic in the world as declared by W.H.O. on March 12, 2020. It is a viral disease caused by SARS-CoV 2 virus and has affected large populations in 231 countries. There is no specific treatment available and management is empirical. On the backdrop of this novel corona virus (COVID-19-SARS-CoV2) creating a pandemic and resulting in lockdown of cities with sealing of the borders, it is an emergent need that the medical and scientific community undertake quick measures. This virus has made social, political, economic, financial, medical, and scientific fraternity to go in a state of emergency. The spread of the virus has been as fast as the dissemination of fear and false news. Rapid isolation measures (test, trace and treat), social distancing of two meters, hand washing with soap and water for 20 seconds or sanitizer and wearing of masks have restricted the spread. Yet, we still have not found solutions to treat the COVID 19 disease when it has affected individuals despite many drug and vaccine clinical trials that the world is looking forward to. We have also not been able to device methodology to prevent the occurrence of infection, to find drugs that can act as prophylactic medicines or drugs that can prevent the affected patient from developing severe lung injury and other complications that can ensue. Present commentary deals with some of these prophylactic measures.

Keywords: CoVid 19, Prophylaxis, Repurposing drugs

*Corresponding Author Email: drmapaa@yahoo.com

Received 05 July 2020, Accepted 15 July 2020

Please cite this article as: Phadke M et al., COVID19 and Repurposing of Drugs for Prophylaxis

British Journal of Medical and Health Research 2020. 


\section{INTRODUCTION}

Coronavirus belongs to the family of coronaviridae in the order Nidovirales. There are four groups of coronaviruses ${ }^{1}$ alphacoronaviruses infect mammals (human coronavirus NL63), Betacoronaviruses infect mammals (SARS CoV, MERS CoV, Bat CoV), gammacoronaviruses infect birds (avian infectious bronchitis coronavirus), deltacoronaviruses infect both birds and mammals (porcein delta CoV). COVID19 is a betacoronavirus ${ }^{1}$. Six species of human coronaviruses are known, with one species subdivided into two different strains, making seven strains of human coronaviruses altogether.

Three human coronaviruses produce symptoms that are potentially severe:

1. Middle East respiratory syndrome-related coronavirus (MERS-CoV), $\beta-\mathrm{CoV}$

2. Severe acute respiratory syndrome coronavirus (SARS-CoV), $\beta-\mathrm{CoV}$

3. Severe acute respiratory syndrome coronavirus 2 (SARS-CoV-2), $\beta-\mathrm{CoV}$

Genetic mutations occur in the virus and are a natural phenomenon. During the evolution of the pandemic, 3 strains of mutations spread across the globe, having originated probably from Wuhan, China. A, B and C are the mutations. Type A has been found mainly in the US and Australia, B strain in East Asia, C strain is mutation from B and seen in Europe (not China).C strain is also the one in Hongkong, Singapore, S. Korea from where it went to Europe. USA and UK have detected G strain. Chinese scientists have labeled 2 strains that they found, L strain (70\%) which is more aggressive and found early, and S strain which is less aggressive and comprised $30 \%$. As per a study from West Bengal ${ }^{2}$ the original strain of the COVID-19 causing virus was named $O$ type or ancestral type. But from this strain at least 10 different strains had originated. Out of these 10 new strains, a strain called A2a is currently the most prevalent in the world as well as in India. The study claims that if a vaccine is to be made, it should be made against this strain. The A2a strain reportedly has an advantage over its peers in that it can better bind with the ACE-2 receptors in the lungs - which is what the SARS-CoV2 virus uses to enter healthy cells.

It was on February 11, 2020, that the international committee on taxonomy of viruses announced severe acute respiratory syndrome corona virus 2 as the name of this new virus earlier called novel corona virus (nCov). It is a large (27-32 kb), enveloped, positive stranded RNA virus. Its viral genome is packed inside a helical capsid formed by nucleocapsid protein and is surrounded by an envelope. The virus has characteristic club-shaped spikes that project from its surface, which in electron micrographs create an image reminiscent of the solar corona, from which the name derives. ${ }^{3}$

The virus has four proteins: membrane protein $(\mathrm{M})$, envelope protein $(\mathrm{E})$, spike protein $(\mathrm{S})$, and nucleocapsid protein $(\mathrm{N})$. Spike forms large protrusions on viral surface. Spike protein 
mediates the entry of the virus into host cells ${ }^{4}$. It is also the critical determinant of viral host range and tissue tropism and is a major inducer of host immune response ${ }^{3}$. Spike protein oversees receptor binding and subsequent viral entry into the host cells. Therefore, it can be the major therapeutic target. It binds to the cellular receptor angiotensin converting enzyme 2 (ACE2) and cellular receptor dipeptidyl peptidase 4 (DPP 4) or CD 26. The former is more for SARS and the latter is for MERS.

There is no definite treatment for COVID-19. The most important method is to prevent viral transmission by rapid isolation and disease containment measures. Treatment of pneumonia and of acute lung injury is largely empiric. There is an urgent need to device and develop various treatment modalities in the form of vaccine, newer drug molecules, or repurpose some of the existing drugs that can kill the virus and prevent the cytokine storm with development of pneumonia and ARDS like lung injury. As of date two important international drug trials RECOVERY (arms-Ritonavir, Lopinavir, Hydroxychloroquine, Dexamethazone, and plasma) and SOLIDARITY (arms-Remdesivir, Lopinavir, Ritonavir, Hydroxychloquine) are being undertaken besides many others.

Given these facts about the CoVid 19 scenario, there appears to be an equally urgent need to device newer methods as under:

1. Prevention of SARS CoV2 viral entry -Physical methods, barriers.

2. Prevention of entry of SARS CoV2 virus in human body-Immunological boosting, vaccines

3. Prevention of entry of the SARS CoV2 virus by local chemical methods

4. Prophylaxis for arresting the viral multiplication in human host by drugs

5. Prophylaxis to prevent lung injury.

\section{Prevention of SARS CoV2 viral entry -Physical methods, barriers.}

These are all the methods adopted by the world like social distancing of two meters, repeated hand washing with soap and water for 20 seconds, sanitizers, wearing masks, head gears, not touching hands to nose mouth and eyes. Lock down of cities, staying at home and sealing borders for domestic and international travel are essential.

\section{Prevention of entry of SARS CoV2 virus in human body.}

Till such time that effective vaccines are made available, communities will rely on their innate immunity. Role of balanced diet and micronutrients is even more important during 'CoVid times. With prevailing lock down in many regions, supply chains of food have got disrupted. Loss of jobs may push people into poverty and may aggravate malnutrition especially in children, adolescent girls, and women. Income elasticity differs for different foods. Thus, for every $1 \%$ drop in income, fat intake decreases by $0.149 \%$, protein by $0.127 \%$ and carbohydrates by $0.122 \%$. The same is true for Iron and Zinc, the fall being 0.135 and 0.130 
$\%$. It is negligible for Sodium (0.012) and Potassium $(0.1)^{5}$. This food inelasticity is observed much more in poor than in the rich population.

CoVid 19 crisis has made it clear that inequality is a maker and marker of malnutrition. In developing countries, it is a health, economic, social and nutrition crisis. It is seen that undernourished people have weak immune system and may get severe CoVid disease. Similarly, those who have poor metabolic health like diabetes and obesity show worse outcomes. Nutritional resilience is very essential for CoVid 19 combating mechanisms. This is the link between public health and equity as nutritional resilience is compromised in weaker sections, women, children, the poorer and marginalized communities. Effect of lockdown will probably result in fall in vitamin D levels in adults and children. In one study nearly $70 \%$ of Indian population is seen to have Vitamin D deficiency. This will be exaggerated during lockdown, particularly so in children, pregnant \& lactating mothers, and senior citizens.

\section{Prevention of entry of the SARS CoV2 virus by local chemical methods.}

SARS CoV2 virus enters the human host through respiratory passages, gastrointestinal route and sometimes conjunctiva. It generally takes one day for the virus cycle and the incubation period is 2-14 days. During this period, the virus enters the cells of epithelia.

Viral entry relies on a fine interplay between the virion and the host cell. Infection is initiated by interaction of the viral particle with specific proteins on the cell surface. After initial binding of the receptor, enveloped viruses need to fuse their envelope with the host cell membrane to deliver their nucleocapsid to the target cell. The spike protein plays a dual role in entry by mediating receptor binding and membrane fusion. The fusion process involves large conformational changes of the spike protein. Corona viruses use a variety of receptors and triggers to activate fusion, however fundamental aspects that enable this initial step of the viral life cycle are conserved. Entry mechanisms focusing on the different trigger are used by corona viruses to initiate the conformational change of the $\mathrm{S}$ protein: receptor binding, low $\mathrm{pH}$ exposure and proteolytic activation. ${ }^{6}$ Thus prevention of viral entry can involve blocking of any of these entry methods including change of $\mathrm{pH}$.

Several antibacterial, antiviral gargles, nasal sprays have been tried. They have short lived action and need to be used every two hours. The solutions available contain Povidone-iodine (PVP-I) which is a broad-spectrum antimicrobial that has been used in infection control and prevention for over 60 years. PVP-I has well-established general antimicrobial activity, demonstrating in vitro efficacy against gram-positive, gram-negative and some spore-forming bacteria (clostridia, Bacillus spp.) and mycobacteria and a wide range of enveloped and nonenveloped viruses. Recent in vitro studies have demonstrated rapid virucidal activity of PVP-I products against Ebola virus, MERS-CoV and European reference enveloped virus [modified vaccinia virus Ankara (MVA) $]^{7}$. Considering the proven in vitro efficacy, gargling with PVP- 
I (Betadine) may be an effective method of preventing the spread of respiratory viruses when an individual is contaminated by the airborne/droplet route or after uptake via the mouth.

\section{Prophylaxis for arresting the viral multiplication in human host by drugs.}

In areas where physical barriers like social distancing is not possible due to high population density in slum dwellers, prophylactic drugs will be useful. These are drugs used for other indications and can be repurposed ${ }^{8}$.

Doxycycline is a broad-spectrum antibiotic with antibacterial, antiviral, and antiprotozoal actions. It has anti-inflammatory properties. Published literature ${ }^{9,10}$ shows that it is effective in prevention of leptospirosis, malaria, chikungunya and now few reports on CoVid 19. Lipophilic nature of doxycycline along with its ability to inhibit matrix metalloproteinases (MMP) helps it to chelate on zinc compound in MMP. Corona virus depends on these MMPs for their survival, infiltration, cell to cell adhesion and replication. So, doxycycline acts as antiviral drug preventing corona virus (SARS Cov2) from multiplication. The drug has been used by medical fraternity in India for several decades. Ivermectin is a deworming drug used for several decades and has in vitro activity against SARS Cov 2. Doxycycline $200 \mathrm{mgs}$. once a week for few months and Ivermectin 3 mgm single dose every week should be used. Dosages can be altered. Doxycycline gargles and sprays should be available.

Hydroxychloroquine (HCQ) is being tried as an antiviral in the treatment of CoVid 19 with mixed results, largely negative. Its role in prophylaxis is yet to be evaluated. Indian council of Medical Research recommends the use of HCQ in prophylaxis. It may have a definite role in view of long half-life of HCQ and a smaller dose, thereby reducing cardiac toxicity.

\section{Prophylaxis to prevent lung injury.}

If the patient has developed the disease, the aim would be to stop lung injury. This is in addition to many antiviral drugs and other supportive empiric therapy.

Interferon beta may have a role. Interferon beta is part of the body's first line of defense against viruses, warning it to expect a viral attack. Corona viruses suppress endogenous IFN-beta production to help the virus affect the innate immune system. Southampton University scientists (UK) have developed interferon beta inhaler. The drug is used in multiple sclerosis and is repurposed for its probable efficacy in preventing CoVid 19 lung injury. It is given as an inhaler spray.

The drug SNG 001 is a special formulation of interferon beta delivered directly to the airways when the virus is there, with the hope that a direct dose of the protein will trigger a stronger anti-viral response even in patients whose immune systems are already weak. The trial is ongoing. ${ }^{11}$ 


\section{BCG vaccine, MMR, and Polio vaccine}

BCG vaccine is an age-old vaccine used for its efficacy in preventing Tuberculosis, specially preventing dissemination. BCG vaccination reacts with the human immune system, suggests it can have general immune-boosting effects. When scientists have compared the immune systems of people who had the BCG vaccine with those who have not, they discovered that the immune cells that first respond to disease in BCG vaccinated people are more alert and ready to act on a potential threat. An alert immune system means the body is better prepared to fight disease. This idea has been supported by studies carried out in babies born in Guinea-Bissau. These suggested that the vaccine may help reduce child mortality beyond what you would expect by preventing TB alone. Reports from trials also suggest it could reduce the likelihood of people getting other respiratory infections, some of which are caused by viruses with a very similar form to COVID-19. Even if it cannot stop people contracting the disease, certain data suggests that there is a chance it may prove helpful in reducing the severity of symptoms responsible for the pandemic death toll. A number of research teams in Australia, Netherlands, India to Germany are taking a closer look at whether receiving a dose of the BCG vaccine may prevent or help people fight off a COVID-19 infection and reduce the number of COVID-19related deaths. A new, BCG vaccine called VPM1002 is being tried in Germany to find out if it can protect health care workers and older patients from COVID-19, lung injury. Mice infected with influenza were also found to have lower levels of influenza viruses in their blood if they were given the BCG vaccine. If successful, this repurposing for CoVid 19 lung injury prophylaxis will be rewarding.

MMR vaccine and prevention of CoVid19. Administering the MMR (measles, mumps, rubella) vaccine could serve as a preventive measure to dampen septic inflammation associated with the CoVid19 virus infection. Studies are underway. ${ }^{13}$

Repurposing oral polio vaccine to give protection against CoVid19 virus, by nonspecific boosting of immunity is being undertaken. All the five prophylactic strategies suggested will testify the truth. Obviously, the jury is still out.

\section{REFERENCES:}

1. Zhu, N., \& Zhang, D. (2020). Wenling Wang, pneumonia in China. The New England Journal of Medicine, 382, 725-733.

2. National Institute of Biomedical Genomics, West Bengal Apr.28.2020

3. Li, F. (2016). Structure function and evolution of corona virus spike proteins. Annual Review of Virology, 3(1), 237-261.

4. Mc Lellan, Daniel Wrapp www.livescience.com, 2020 
5. Raghav Gaiha \& Nidhi Kaicker \& Katsushi S. Imai \& Vani S. Kulkarni \& Ganesh Thapa, 2012. "Has Dietary Transition Slowed Down in India: An Analysis Based on 50th, 61st and 66th Rounds of NSS," Discussion Paper Series DP2012-15, Research Institute for Economics \& Business Administration, Kobe University.

6. Sandrine Belouzard,1 Jean K. Millet,2 Beth N. Licitra,2 and Gary R. Whittaker2, Mechanisms of Coronavirus Cell Entry Mediated by the Viral Spike Protein Viruses. 2012 Jun; 4(6): 1011-1033. Infect Dis Ther. 2018 Jun; 7(2): 249-259.

7. Infect Dis Ther. 2018 Jun; 7(2): 249-259.Published online 2018 Apr 9. doi: 10.1007/s40121-018-0200-7PMCID: PMC5986684 PMID: 29633177,In Vitro Bactericidal and Virucidal Efficacy of Povidone-Iodine Gargle/Mouthwash and against Respiratory Oral Tract Pathogens

8. Mrudula Phadke, Sujata Saunik, COVID-19 treatment by repurposing drugs until the vaccine is in sight Drug Dev Res. 2020;1-3. wileyonlinelibrary.com/journal/ddr

9. Genetic Engineering and Biotechnology newsFeb27,2020, Drug virus.info

10. M. Sodhi, Tetracyclines proposed as treatment for CoVid19, Apr.8.2020 https://accpjournals.onlinelibrary.wiley.com/doi/abs/10.1002/phar.2395 BACK

11. Pharmaphorum; Synairgen doses first patients with COVID-19 drug,31 ${ }^{\text {st }}$ Mar2020.

12. GAVI Can the BCG vaccine protect against CoVid 19? 19th May 2020

13. Gold J. E. MMR Vaccine Appears to Confer Strong Protection from COVID-19: Few Deaths from SARS-CoV-2 in Highly Vaccinated Populations10.13140/RG.2.2. May 202

\section{BJMHR is}

15. Peer reviewed

16. Monthly

17. Rapid publication

18. Submit your next manuscript at

19. editor@bjmhr.com 